\title{
DER SPRINGER COMPACT-DEAL - EIN ERSTER EINBLICK IN DIE EVALUIERUNG EINER OFFSETTING-VEREINBARUNG
}

\author{
von Magdalena Andrae \& Márton Villányi
}

Zusammenfassung: Seit Anfang 2016 gilt zwischen 32 wissenschaftlichen Bibliotheken in Österreich und dem Springer-Verlag eine gänzlich neuartige Vereinbarung, die neben der üblichen Lizenzierung von Inhalten auch die Möglichkeit bietet, in den Springer-Zeitschriften Open Access zu publizieren. Mehr als 1.000 Autorinnen und Autoren aus Österreich haben im ersten Jahr diese Möglichkeit genutzt. Die Daten dazu sind nun auf der Plattform OpenAPC einzusehen. ${ }^{1}$ Ein guter Anlass, eine Zwischenbilanz zur Evaluierung des Deals zu ziehen.

Schlüsselwörter: Österreich; OpenAPC; Publikationskosten; Konsortialvertrag; Transformation zu Open Access

\section{THE SPRINGER COMPACT DEAL - FIRST INSIGHTS INTO THE EVALUATION OF AN OFFSETTING AGREEMENT}

Abstract: On January the 1 $1^{\text {st }}, 2016$ a new agreement between 32 Austrian scientific libraries and the publisher Springer took its effect: this deal covers accessing the licensed content on the one hand, and publishing open access on the other hand. More than 1000 papers by Austrian authors were published open access at Springer in the first year alone. The working group "Springer Compact Evaluierung" made the data for these articles available via the platform OpenAPC and would like to use this opportunity to give a short account of what this publishing agreement actually entails and the working group intends to do.

Keywords: Austria; OpenAPC; article processing charges (APC); consortial agreement; transition to open access

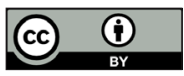

Dieses Werk ist lizenziert unter einer

Creative-Commons-Lizenz Namensnennung 4.0 International 


\section{Inhalt}

1. Der konsortiale Springer-Compact-Deal und seine Evaluierung

2. Die OpenAPC-Initiative und ihr Nutzen für die Evaluierung

3. "Offsetting" und "Read and Publish" auf der Plattform OpenAPC

4. Die Evaluierung des österreichischen Deals - Ein Ausblick

\section{Der konsortiale Springer-Compact-Deal und seine Evaluierung}

Im Jahr 2015 vereinbarte die Kooperation E-Medien Österreich (KEMÖ) in Zusammenarbeit mit dem österreichischen Wissenschaftsfonds (FWF) ein neuartiges Lizenzmodell mit dem Springer Verlag. Aufgrund dieses „Read and Publish"-Deals erhalten Wissenschaftlerinnen und Wissenschaftler in den Jahren 2016 bis 2018 Zugriff auf über 2.000 SpringerZeitschriften sowie gleichzeitig die Möglichkeit, in mehr als 1.600 Subskriptionszeitschriften des Verlags ohne Zusatzkosten Open Access zu publizieren.

Bibliotheken zahlen also dem Verlag eine Gesamtgebühr, die neben der Lizenzierung auch das Publizieren beinhaltet. Dieses neuartige Modell mit dem Namen Springer Compact soll ein weiterer wichtiger Schritt auf dem Weg zur vollständigen Open Access-Transition des wissenschaftlichen Publikationswesens werden.

Inwieweit Springer Compact die Erwartungen der Bibliotheken erfüllen wird, kann im Moment noch nicht beantwortet werden. Nach Ablauf des ersten Vertragsjahres 2016 wurde aber im Rahmen der KEMÖ eine Arbeitsgruppe auf die Beine gestellt, um wesentliche Faktoren wie Publikationsleistung oder Nutzung des Portfolios durch Autorinnen und Autoren genauer unter die Lupe zu nehmen. Die hier gewonnenen Erkenntnisse sollen Vor- und Nachteile eines solchen Deals aufzeigen und dem Konsortium Unterstützung bei zukünftigen Verhandlungen bieten. Ähnliche Evaluierungen wurden auch in anderen Ländern mit vergleichbaren Verträgen initiiert: Großbritannien, Schweden, die Niederlande und die MPDL (Max Planck Digital Library) analysieren ebenso ihre Springer-Compact-Vereinbarungen. Fragen, die im Zuge dessen beantwortet werden sollen, sind beispielsweise:

- Welche Auswirkungen hat der Deal auf das Publikationsaufkommen?

- Wie verändert sich die Nutzung der lizenzierten Inhalte?

- Taugt eine solche Vereinbarung als Motor zur Open Access-Wende? 
Erste diesbezügliche Erkenntnisse sollen Anfang des Jahres 2018 vorliegen.

Für eine fundierte Analyse des österreichischen Publikationsaufkommens bedarf es jedoch einer konsistenten Datengrundlage. Um diese möglichst transparent und auch den Evaluierungsteams anderer Länder zugänglich zu machen, beschloss die österreichische Arbeitsgruppe in einem ersten Schritt dem Beispiel anderer Länder zu folgen und die bereinigten Publikationsdaten des Konsortiums über die Plattform OpenAPC zur Verfügung zu stellen.

\section{Die OpenAPC-Initiative und ihr Nutzen für die Evaluierung}

Die Darstellung des Publikationsvolumens von Open Access-Deals ist nur ein kleiner, wenn auch nicht unwesentlicher Teil der OpenAPC-Plattform. Wie der Name verrät, ist die ursprüngliche Intention der Initiative, Daten zu APC-Zahlungen - also Open Access-Publikationsgebühren in Gold- und Hybrid Open-Access-Zeitschriften - zu sammeln. Eingebettet ist die Initiative in das von der deutschen Forschungsgemeinschaft (DFG) finanzierte Projekt INTACT, das von der UB Bielefeld, dem Institute for Interdisciplinary Studies of Science der Universität Bielefeld (I2SoS) und der Max Planck Digital Library (MPDL) getragen wird und dessen Ziel die Schaffung einer transparenten Infrastruktur für Open Access-Publikationskosten ist. Neben OpenAPC finden sich auch die Initiativen ESAC (Efficiency and Standards for Article Charges) und OA Analytics (Statistics on Open Access Publishing) im Rahmen dieses Projekts. Die Sammlung von empirischen Daten zu den Kosten von Open Access im Zuge von OpenAPC dient damit in einem breiteren Kontext der Unterstützung der Kostenanalyse sowie dem Ziel, standardisierte Berichts- und Geschäftsprozesse zu ermöglichen. Praktisch umgesetzt wird OpenAPC über Github. ${ }^{2}$ Github Inc. ist eine HostingPlattform, die u.a. für die Software-Weiterentwicklung bei OpenSourceProjekten genutzt werden kann. Im Fall von OpenAPC ist durch Github für die einzelnen beteiligten Institutionen ein offener und transparenter Weg geschaffen worden, um Daten über die Ausgaben für Open Access zentral abliefern und damit in das Gesamtprojekt speisen zu können. Auch die Aufbereitung und Weiterverarbeitung der Daten erfolgt über Github.

Die Plattform bietet also einerseits Rohdaten zu Open Access-Publikationen, für die eine Gebühr entrichtet wurde; diese können heruntergeladen und in späterer Folge analysiert, angereichert und nachgenutzt werden. Anderseits werden die Daten auch direkt auf der Plattform grafisch aufbereitet; diese Visualisierungen geben einen guten ersten Einblick über Publikationsaufkommen und -ausgaben einzelner Einrichtungen, Verlage, Jahre etc. 


\section{3. „Offsetting“ und „Read and Publish“ auf der Plattform OpenAPC}

Innerhalb der Plattform finden sich die Springer Compact-Daten mehrerer Länder im separaten Bereich „Offsetting“. Diese Abgrenzung war notwendig, da in diesem Fall für Publikationen keine einzelnen APCs mehr entrichtet werden, sondern in einem „Read and Publish"-Deal mitinbegriffen sind.

Im Open Access-Kontext decken die Begriffe „Offsetting“ und „Read and Publish“ ähnliche, wenn auch nicht idente Aspekte ab: Beim „Offsetting“Modell versuchen Lizenznehmende (Bibliotheken, Konsorten) und Lizenzgebende (Verlage) eine doppelte Zahlung, das sog. das "Double dipping“, zu vermeiden. Dies kann auftreten, wenn erstens die Einrichtungen für ihre Zeitschriftenpakete eine Lizenzgebühr entrichten und zweitens Forschende derselben Einrichtung in diesen Zeitschriften gegen Bezahlung Open Access publizieren. Der Verlag kassiert also in solchen Fällen doppelt; einerseits von den Bibliotheken und andererseits von den Autorinnen und Autoren. Um dem entgegenzuwirken, sollen im jeweils folgenden Jahr diese Ausgaben den Lizenzgebühren gutgeschrieben werden.

Dies erfordert allerdings einen sehr hohen administrativen Aufwand, sodass das „Read and Publish“-Modell durchaus auch als Reaktion auf das mit dem „Offsetting“ verbundene umständliche Prozedere verstanden werden kann. Dabei zahlt die Einrichtung einmalig für beide Komponenten. Das hat zur Folge, dass sowohl der Zugriff auf das gesamte Zeitschriftenportfolio gewährleistet wird als auch die Forschenden ohne Mehrkosten und -aufwand Open Access publizieren können. Springer nimmt in diesem Zusammenhang eine Vorreiterrolle ein. Weitere Verlage, die dieses Open Access-Geschäftsmodell ebenfalls bereits verfolgen, sind beispielsweise Emerald und Wiley-Blackwell.

Übereinkünfte zur Gegenverechnung von Open Access-Gebühren, die Gegenstand beider Modelle sind, stellen für die OpenAPC-Plattform eine Herausforderung dar, da die Bepreisung einzelner Artikel nicht mehr so eindeutig durchgeführt werden kann. Publikationen, die auf solchen Wegen Open Access publiziert wurden, sollten jedoch genauso erfasst werden - stellen doch diese Deals ein wichtiges Mittel zur Transformation hin zu Open Access dar. Nur so lässt sich ein breites Bild des Open AccessMarktes, mit all seinen Facetten, erstellen.

Zur Zeit sind in den „Offsetting“-Datensätzen keine Preisinformationen enthalten, da die entstandenen Kosten noch nicht auf die einzelnen Artikel heruntergebrochen werden können. Ident zu der restlichen Datenbank werden jedoch Verlag, Zeitschrift, DOls und die Zugehörigkeit der korrespondierenden Autorinnen und Autoren angeführt. ${ }^{3}$ 
Zunächst beinhaltete der „Offsetting“-Bereich der OpenAPC-Plattform Daten zu Springer Compact-Publikationen aus Deutschland (Max PlanckGesellschaft), Großbritannien, Niederlande, Österreich und Schweden. Während der Fertigstellung dieses Artikels wurden jedoch bereits die ersten Daten zu „Offsetting“-Deals mit Taylor \& Francis und der Royal Society of Chemistry (RSC) durch die Max Planck-Gesellschaft hochgeladen ${ }^{4}$; weitere Datenlieferungen - z.B. durch die Kooperation E-Medien Österreich (KEMÖ) - könnten diesen Datenbestand in näherer Zukunft noch anwachsen lassen.

Da die Springer Compact-Daten im Unterschied zu den eigentlichen APC-Meldungen von institutsübergreifenden Organisationen wie Konsortien oder Arbeitsgruppen zur Verfügung gestellt wurden, ist auch von einer Vollständigkeit auszugehen. Es wird hier nicht ein Sample von kooperierenden Einrichtungen sichtbar, sondern der flächendeckende Publikationsoutput ganzer Konsortien. Dies ermöglicht in späterer Folge nicht nur einen Vergleich über die Jahre, sondern auch zwischen den Verlagen, und dies sowohl auf nationaler als auch internationaler Ebene. Die Attraktivität unterschiedlichster Deals wird dadurch einfacher quantifizierbar.

Über die Plattform lassen sich verschiedenste Aspekte darstellen, wie z.B.:

- Von welchen Einrichtungen kommen die Autorinnen und Autoren?

- In welchen Zeitschriften wird bevorzugt publiziert?

- Wie steht Österreich im internationalen Vergleich da?

Sobald diese Zahlen zu anderen Quellen in Relation gesetzt werden, können außerdem weitere wertvolle Erkenntnisse gewonnen werden.

Die Bereitstellung der Daten eines gesamten Konsortiums stellt für Österreich jedenfalls ein Novum dar. Auf Basis der einzelnen APC-Zahlungen ist die Initiative jedoch auf die Kooperation der einzelnen Einrichtungen angewiesen. Außer dem Springer-Konsortium wurden hierzulande bisher nur die Daten des österreichischen Wissenschaftsfonds (FWF) ${ }^{5}$, der Technischen Universität Wien ${ }^{6}$ und des Institute of Science and Technology Austria $^{7}$ veröffentlicht. Um aussagekräftige Kostenanalysen und Prognosen durchführen zu können und eine offene Diskussion über alternative Publikationsmodelle anzustoßen, sollten noch viele weitere österreichische Einrichtungen deren Beispiel folgen.

\section{Die Evaluierung des österreichischen Deals - Ein Ausblick}

Die Offenlegung der Daten auf OpenAPC stand zunächst nicht im Fokus der Arbeitsgruppe „Evaluierung Springer Compact“. Im Rahmen der ersten 
Treffen stellte sich jedoch rasch heraus, dass die Betrachtung des Vertrags in einem breiteren Kontext - konkret in Relation zu anderen Springer Compact-Verträgen - durchaus Sinn machen würde. Als daraufhin klar wurde, dass die AG nicht nur das vorliegende „Read and Publish“-Modell auswerten würde, sondern auch die Erarbeitung von Leitlinien für die Bewertung weiterer Open Access-Verlagsvereinbarungen Ziel sein sollte, wurde auch die Teilnahme an OpenAPC beschlossen.

Die eigentliche Aufgabe der Arbeitsgruppe besteht in der Formulierung von aussagekräftigen Evaluierungskriterien einerseits und der Auswertung der Daten aus dem Jahr 2016 andererseits.

Bei der Formulierung besagter Kriterien konnte zum Teil auf die Vorarbeit anderer Länder zurückgegriffen werden. Spezifische österreichische Bedingungen mussten jedoch berücksichtigt werden. Außerdem sollten die Untersuchungsmethoden auch in späteren Jahren möglichst nachnutzbar sein. Soweit möglich war auch die Übertragbarkeit des Kriterienkatalogs auf zukünftige Deals erstrebenswert.

In einem weiteren Schritt müssen die vorhandenen Daten über das Publikationsaufkommen und/oder die Nutzung der Zeitschriften einer Kontrolle unterzogen werden, um die eigentliche Arbeit, nämlich die Analyse durchführen zu können. Dieser wird gegenwärtig in zwei spezialisierten Arbeitsgruppen nachgegangen. Erste Teilergebnisse werden beim Österreichischen Bibliothekartag im September 2017 präsentiert. Mit einem umfangreichen Bericht ist mit Anfang 2018 zu rechnen. Open Access-Deals zwischen Bibliotheken und Verlagen werden in den nächsten Jahren vermutlich statt der Ausnahme zum Standard werden, und so ist Arbeitsgruppe zur Evaluierung von Springer Compact zuversichtlich, mit ihren Analysen eine Hilfestellung bei dieser Entwicklung bieten zu können.

MMag. ${ }^{a}$ Magdalena Andrae Technische Universität Wien, Universitätsbibliothek E-Mail: magdalena.andrae@tuwien.ac.at

Mag. Márton Villányi Institute of Science and Technology Austria (IST), Library E-Mail: marton.villanyi@ist.ac.at 
1 https://treemaps.intact-project.org/ [Stand 27.07.2017]

2 https://github.com/OpenAPC/openapc-de [Stand 27.07.2017]

3 https://treemaps.intact-project.org/apcdata/offsetting/ [Stand 27.07.2017]

4 Einsehbar unter: https://github.com/OpenAPC/openapc-de/tree/master/ data/offsetting/mpg [Stand 27.07.2017]

5 https://treemaps.intact-project.org/apcdata/fwf/ [Stand 27.07.2017]

6 https://treemaps.intact-project.org/apcdata/tu-wien/ [Stand 27.07.2017]

7 https://treemaps.intact-project.org/apcdata/ist-austria/ [Stand 27.07.2017] 